\title{
Análisis de las competencias y conocimientos en atención a la dependencia en Trabajo Social en España
}

\author{
Enrique Pastor Seller* \\ Ana García-Estañ Forn ** \\ Recibido: 18 octubre de 2016 \\ Enviado a pares evaluadores: 25 de octubre de 2016 \\ Aprobado por pares evaluadores: 17 de noviembre de 2016 \\ Aprobado por Comité Editorial: 24 de noviembre de 2016 \\ DOI: $10.22395 /$ csye.v5n10a2
}

\section{RESUMEN}

El artículo presenta los resultados de una investigación orientada a identificar, describir y analizar las competencias y conocimientos en materia de atención a personas en situación de dependencia que integra la Enseñanza Superior de grado en Trabajo Social en España. El artículo presenta un estudio del universo de los títulos de grado en Trabajo Social que se imparte en 39 universidades españolas. Este acercamiento permite identificar los 32 centros universitarios que integran en sus planes de estudio la materia de dependencia, analizando las competencias específicas que establecen cada una de las asignaturas. Asimismo, se han identificado y analizado, a través de las guías docentes, las 43 asignaturas vinculadas con dependencia y las 216 competencias específicas. El análisis de contenido ha conllevado la identificación y descripción final de 93 competencias integradas en tipologías atendiendo a las funciones profesionales. Los resultados alcanzados permiten disponer de un conocimiento acerca de la cobertura general de la titulación de Grado en Trabajo Social en España, la estructura y organización docente de las asignaturas vinculadas directamente con la dependencia y las competencias que establecen las asignaturas específicas de dependencia relacionándolas con la actividad profesional. Del análisis se constata, entre otros resultados, que la mayoría de las asignaturas de dependencia identifican competencias muy genéricas, la existencia de evidentes divergencias en el tratamiento de la enseñanza de la dependencia en las diferentes universidades españolas y la necesidad de una mayor adaptación/ actualización de la enseñanza de la dependencia a las necesidades profesionales y del entorno laboral.

Palabras clave: Trabajo social; educación superior; competencias; habilidades; dependencia.

\footnotetext{
* Doctor en Sociología y Política Social. Profesor titular del Departamento de Trabajo Social y Servicios Sociales de la Universidad de Murcia. Director Grupo Investigación Trabajo Social y Servicios Sociales. Correo electrónico: epastor@um.es

** Graduada en Trabajo Social. Trabajadora social en Ayuntamiento de Murcia (Murcia). España. Correo electrónico: ana.garcia@ayto-murcia.es
} 


\section{Analysis of competences and knowledge in attention to the dependence in Social Work in Spain}

\section{ABSTRACT}

The article presents the results of an investigation aimed at identifying, describing and analyzing the skills and knowledge in care of people in a situation of reliance that integrates higher education of degree in Social Work in Spain. The article presents a study of the universe of degrees in Social Work that is taught in 39 Spanish universities. This approach allows identifying the 32 university centers that integrate the subject of dependency in their curricular, analyzing the specific competences that establish each one of the subjects. Likewise, the 43 subjects related to dependency and the 216 specific competences have been identified and analyzed, through the teaching guides. The analysis of content has entailed the identification and final description of 93 competences integrated in typologies according to the professional functions. The results obtained allow to have a knowledge about the general coverage of the Degree in Social Work in Spain, the structure and teaching organization of the subjects linked directly to the dependency and the competences that establish the specific subjects of reliance relating them to the professional activity. From the analysis we can see, among other results, that most of the subjects of reliance identify very generic competences, the existence of evident divergences in the treatment of the teaching of the dependency in the different Spanish universities and the necessity of a greater adaptation/update. Of the teaching of the dependency to the professional needs and of the working environment.

Keywords: Social Work; higher education; competences; skills; dependence. 


\section{Introducción}

La creación del Espacio Europeo de Educación Superior (en adelante EES) ha supuesto para todos los países involucrados la adopción de una nueva organización de los estudios universitarios oficiales, entre otros, "un sistema basado esencialmente en dos ciclos formativos: grado (de clara orientación al mercado de trabajo) y posgrado" (Vázquez, 2005a, p. 240) y en la formación en competencias o la formación para desarrollar la capacidad de actuación con los conocimientos adquiridos. A partir de 2007 en España, el grado en Trabajo Social se adapta a las EEE y en el curso académico 2008/2009 se inició el proceso de implantación de los planes de estudios al nuevo marco en todas las universidades españolas. Este nuevo marco supuso para los estudios de trabajo social el acceso a estudios de postgrado y doctorado, así como la equiparación de los estudios respecto del resto de titulaciones universitarias en un marco de igualdad.

En los últimos años ha existido una mayor formación de los trabajadores sociales en diferentes ámbitos de actuación, como es el judicial, la violencia de género, el sanitario, la educación y el que nos ocupa, la dependencia. La enseñanza en Trabajo Social en las universidades ha incorporado las implicaciones que ha supuesto la Ley 39/2006 de 14 de diciembre, de Promoción de la Autonomía Personal y Atención a las personas en situación de dependencia, concretamente el reconocimiento de derechos hacia las personas en situación de dependencia. La promulgación de la citada ley ha supuesto la emergencia de un sistema de protección específico, la implantación/transformación de centros, servicios y programas y el impulso de un sector propio de intervención profesional. Este nuevo contexto de oportunidades precisa que futuros profesionales del Trabajo Social adquieran competencias, habilidades y conocimientos específicos en materia de dependencia en sus diferentes perspectivas: política, normativa, metodología, valoración, prevención, promoción, programación, intervención, evaluación, etc.

La Ley 39/2006 de 14 de diciembre, de Promoción de la Autonomía Personal y Atención, marco referencial que sirve de orientación para la presente investigación, crea el Sistema de Autonomía y Atención a la Dependencia (en adelante SAAD) definido como el conjunto de servicios y prestaciones económicas destinado a la promoción de la autonomía personal, la atención y protección a las personas en situación de dependencia, a través de servicios públicos y privados concertados debidamente acreditados, y contribuye a la mejora de las condiciones de vida de los ciudadanos. Por ello, el Sistema se configura como una red de utilización pública que integra, de forma coordinada, centros y servicios, públicos y privados. Así mismo, en su artículo 2 define la dependencia como

[...] el estado de carácter permanente en que se encuentran las personas que, por razones derivadas de la edad, la enfermedad o la discapacidad, y ligadas a la falta o a 
la pérdida de autonomía física, mental, intelectual o sensorial, precisan de la atención de otra u otras personas o ayudas importantes para realizar actividades básicas de la vida diaria o, en el caso de las personas con discapacidad intelectual o enfermedad mental, de otros apoyos para su autonomía personal (Ley 39/2006 de 14 diciembre, p. 44144 ).

Es importante la labor del profesional del Trabajo Social en el proceso del $\mathrm{SAAD}$, desde que la persona solicita el reconocimiento de grado, la elaboración del Proyecto de Intervención, la gestión de prestaciones y servicios, seguimiento y evaluación del mismo.

El nuevo marco universitario centrado en la formación en competencias ha supuesto una concepción de la universidad centrada en el aprendizaje (aprendizaje basado en competencias), "cuya finalidad es que los estudiantes lleguen a ser autónomos para aprender determinadas competencias específicas y genéricas de acuerdo con las demandas de la sociedad del siglo XXI" (Rodríguez González, 2006, p. 90). Las competencias, como indican De Juanas y Fernández (2008), en el ámbito académico hacen referencia a aquellas que demanda el desempeño de una profesión. La formación por competencias "supone unos procesos de enseñanza-aprendizaje que transfieren conocimientos, habilidades y actitudes, que capacitan a los futuros profesionales para afrontar las demandas de los contextos laborales y sociales en los que se insertarán" (Gómez, 2010, p. 52).

No olvidemos que "la enseñanza basada en competencias proporciona al alumnado nuevas formas de adquirir las destrezas profesionales y al profesorado de nuevos retos metodológicos de enseñanza en el aula y en el entorno social inmediato" (Pastor-Seller, 2014, p. 179). Una nueva Educación Superior donde el alumnado "es el protagonista de su avance en la interiorización, tanto de contenidos y conceptos, como de la adquisición de competencias, habilidades y aptitudes que lo capaciten para la futura práctica profesional" (Caparrós y Raya, 2014, p. 1134).

Las competencias específicas del Título de Trabajo Social conforme se indican en el Libro blanco y se relacionan con las siguientes para: trabajar y valorar de manera conjunta con personas, familias, grupos, organizaciones y comunidades sus necesidades y circunstancias; planificar, implementar, revisar y evaluar la práctica del trabajo social con personas, familias, grupos, organizaciones, comunidades y con otros profesionales; apoyar a las personas para que sean capaces de manifestar las necesidades, puntos de vista y circunstancias; actuar en la resolución de las situaciones de riesgo con las personas, así como para las propias y las de las colegas de profesión; y administrar y ser responsable, con supervisión y apoyo, de la propia práctica dentro de la organización (Vázquez, 2005b, pp.189-192). 
La adquisición de las competencias, como señala Echeverría (2005), hay que plantearla desde un enfoque holístico, concibiéndolas como el conjunto de conocimientos, procedimientos, capacidades y actitudes, complementarios entre sí de tal forma que los profesionales han de saber, saber hacer, saber estar y saber ser para actuar con la máxima eficacia y eficiencia en sus actuaciones profesionales. De esta forma, las competencias "facilitan el desarrollo de una educación integral ya que engloban todas las dimensiones del ser humano: saber, saber hacer y saber ser y estar" (Rodríguez, 2015, p. 17). Por tanto, conocer las competencias en materia de dependencia es necesario para garantizar una adecuada formación de los futuros profesionales, lo que constituye el objeto de este trabajo. Su finalidad es analizar las competencias que cada universidad establece en sus asignaturas vinculadas directamente con la dependencia y elaborar una síntesis de competencias a modo de tipología, establecidas en cuanto a la especificidad del trabajo social con dependencia.

\section{Método}

La investigación tiene como objetivo general analizar las competencias y conocimientos en materia de atención a personas en situación de dependencia que integran la enseñanza superior de grado en Trabajo Social en España. Para su consecución se proponen los siguientes objetivos específicos:

1) Identificar los centros docentes donde se imparte la Titulación de Grado en Trabajo Social.

2) Identificar y caracterizar las diferentes asignaturas relacionadas con la materia de dependencia de cada una de las titulaciones de Grado en Trabajo Social.

3) Relacionar y clasificar las competencias de las asignaturas relacionadas con la materia de dependencia elaborando tipologías vinculadas con competencias profesionales.

Las variables de estudio, atendiendo a las dimensiones de los objetivos planteados anteriormente, son:

- Cobertura general de la titulación de Grado en Trabajo Social en España.

- Estructura y organización de las asignaturas vinculadas con la dependencia: denominación, integración en itinerario formativo y duración en ECTS ${ }^{1}$.

1 Recordemos un crédito ECTS equivale a 25-30 horas de trabajo: clases lectivas, teóricas o prácticas; estudio personal; participación en seminarios, trabajos, prácticas o proyectos; preparación y realización de exámenes y pruebas de evaluación. 
- Competencias específicas que establece cada título en la/s asignatura/s relacionada/s directamente de dependencia. A través de las guías docentes se recogen las competencias específicas, objetivos y/o resultados de aprendizaje.

\section{Perfil de los centros docentes objeto de estudio}

El Sistema Universitario Español lo conforma un total de 83 universidades (imparten docencia 81), de las cuales 50 son públicas y 33 privadas, (Ministerio de Educación, Cultura y Deporte, 2015, p. 5).

En esta investigación se analizan las universidades españolas donde se imparte el Título de Grado en Trabajo Social, independientemente de su carácter público o privado, centro propio o no. En relación con la búsqueda inicial de cada centro docente donde se imparte el Título se ha utilizado como fuente la web oficial de la Conferencia de Decanos/as y Directores/as de Trabajo Social de la Universidad Española que identifica de manera actualizada el listado de universidades donde se imparte el título, información que ha sido contrastada con una búsqueda actualizada en las distintas webs oficiales de cada una de las universidades en España.

\section{Perfil de las asignaturas objeto de estudio}

Una vez identificado el listado de las universidades donde se imparte el Título de Grado en Trabajo Social se procede a identificar y analizar a través de las guías docentes del curso académico 2015/2016 las asignaturas aquellas que en su definición o denominación integran la categoría de "dependencia" y que tienen relación y contenido directo con el tema de la dependencia, entendiendo que se trate la dependencia de manera transversal y específica en todos sus colectivos (personas mayores, discapacitadas, etc., y no diferenciándose éstos en diferentes asignaturas). Las unidades de análisis son, por consiguiente, las asignaturas cuya denominación integra el concepto explicito de "dependencia". Ello no significa que hayan otras asignaturas en la titulación que aborden la dependencia, pero no de forma transversal e integrada, como pretende la presente investigación.

Por otra parte, la elección del documento de "guía docente" para el análisis de competencias y conocimientos viene argumentado en razón de a) ser el documento público aprobado por los órganos de gobierno de las universidades en el período del trabajo de investigación; b) presentar una descripción detallada del contenido, competencias y conocimientos de las asignaturas, c) identificar su integración en el itinerario formativo y carácter (obligatorio u optativo). La guía docente, como señala Zabalza (2004), es el recurso que el alumnado dispone 
para informarse y orientarse en su aprendizaje. Constituye una herramienta básica del Sistema Europeo de Transferencia de Créditos (ECTS) ${ }^{2}$ para alcanzar la calidad mediante el desarrollo de metodologías y criterios comparables (declaración de Bolonia). Además "es un instrumento de transparencia, fácilmente comprensible y comparable, entre las diferentes universidades en el camino hacia la convergencia" (Universidad de Alicante, 2010, p. 3).

Se han sistematizado las competencias específicas, relacionándolas con las descritas en los dos documentos de referencia normativa, académica y profesional en la Educación Superior en Trabajo Social en España, concretamente el Libro blanco del título de grado de trabajo social (Agencia Nacional de Evaluación y Acreditación -en adelante Aneca-, 2004) y "Criterios para el diseño de planes de estudios de títulos de Grado en Trabajo Social" (Conferencia de Directores y Directoras de Centros y Departamentos de Trabajo Social y Consejo General de Trabajo Social, 2007). La sistematización ha dado como fruto la elaboración de clasificación por tipologías vinculadas con competencias profesionales. En este sentido cabe señalar que algunos centros docentes utilizan diferente nomenclatura: competencias, resultados de aprendizaje y/u objetivos.

La elección de las técnicas e instrumentos de investigación obedece a los intereses de este. Respecto a las técnicas de registro de datos se ha elaborado un protocolo de recogida sistemático de datos ad hoc, realizando una prueba previa de validación del instrumento en dos universidades con el fin de comprobar su coherencia y validez con los objetivos previstos de la investigación.

En cuanto a las técnicas de obtención y sistematización de datos, se han hecho revisiones bibliográficas y webs de instituciones oficiales, con finalidad de obtener datos e información sobre documentos primarios objeto de investigación. Para el análisis de datos se ha utilizado la aplicación informática SPSS 22 (Sistema Global para el análisis de datos) y hoja de cálculo EXCEL, lo que ha permitido sistematizar los mismos y analizarlos por cada universidad con el objeto de obtener los resultados finales. Las categorías competenciales vinculadas con la profesión han sido fruto del análisis de contenido de las competencias identificadas en las guías docentes.

\section{Resultados}

\section{Cobertura de la titulación de Grado en Trabajo Social en España}

En el análisis de la titulación de Grado en Trabajo Social se ha identificado un total de 39 universidades españolas (de las 83 existentes) donde se imparte la

2 Recordemos un crédito ECTS equivale a 25-30 horas de trabajo: clases lectivas, teóricas o prácticas; estudio personal; participación en seminarios, trabajos, prácticas o proyectos; preparación y realización de exámenes y pruebas de evaluación 
titulación de Grado en Trabajo Social, lo que representa el 48,15\% de universidades españolas. En la siguiente tabla (1) pueden identificarse las 39 universidades (públicas y privadas) y sus respectivos centros directivos donde se imparte la titulación de Grado en Trabajo Social.

Tabla 1. Centros docentes de impartición del título de grado en Trabajo Social

\begin{tabular}{|c|c|c|}
\hline \multicolumn{2}{|r|}{$\begin{array}{c}\text { Universidades } \\
\text { Con título de grado en trabajo social }\end{array}$} & Centro directivo \\
\hline 1 & Universidad de Murcia & Facultad de Trabajo Social \\
\hline 2 & Universidad de Cádiz -Jerez- & Facultad de Ciencias del Trabajo \\
\hline 3 & Universidad de Granada & Facultad de Trabajo Social \\
\hline 4 & Universidad de Huelva & Facultad de Trabajo Social \\
\hline 5 & Universidad de Jaén & Facultad de Trabajo Social \\
\hline 6 & Universidad de Málaga & $\begin{array}{l}\text { Facultad de Estudios Sociales y del } \\
\text { Trabajo }\end{array}$ \\
\hline 7 & Universidad Pablo de Olavide -Sevilla- & Facultad de Ciencias Sociales \\
\hline 8 & Universidad de Zaragoza & $\begin{array}{l}\text { Facultad de Ciencias Sociales y del } \\
\text { Trabajo }\end{array}$ \\
\hline 9 & Universidad de Alicante & $\begin{array}{l}\text { Facultad de Ciencias Económicas y } \\
\text { Empresariales }\end{array}$ \\
\hline 10 & Universidad de Almería & $\begin{array}{l}\text { Centro Universitario Adscrito de Trabajo } \\
\text { Social }\end{array}$ \\
\hline 11 & Centro Universitario la Salle -Madrid- & Centro Universitario la Salle \\
\hline 12 & Universidad de Barcelona & Facultad de Educación \\
\hline 13 & $\begin{array}{l}\text { Universidad de Castilla la Mancha - } \\
\text { Campus de Cuenca- }\end{array}$ & Facultad de Trabajo Social \\
\hline 14 & $\begin{array}{l}\text { Universidad de Castilla la Mancha - } \\
\text { Campus de Talavera de la Reina- }\end{array}$ & Facultad de Ciencias Sociales \\
\hline 15 & Universidad Complutense de Madrid & Facultad de Trabajo Social \\
\hline 16 & $\begin{array}{l}\text { * Universidad de Deusto-Campus de } \\
\text { Bilbao- }\end{array}$ & Facultad de Ciencias Sociales y Humanas \\
\hline 17 & $\begin{array}{l}\text { * Universidad de Deusto -Campus de San } \\
\text { Sebastián- }\end{array}$ & Facultad de Ciencias Sociales y Humanas \\
\hline 18 & $\begin{array}{l}\text { * Centro Universitario Santa Ana - } \\
\text { Badajoz- }\end{array}$ & $\begin{array}{l}\text { Centro Universitario. Adscrito a la } \\
\text { Universidad de Extremadura }\end{array}$ \\
\hline 19 & Universidad de Girona & Facultad de Educación y Psicología \\
\hline 20 & Universidad de Islas Baleares & Facultad de Filosofía y Letras \\
\hline 21 & $\begin{array}{l}\text { * Universidad Internacional de la Rioja } \\
\text {-Unir- }\end{array}$ & Facultad de Derecho \\
\hline 22 & Universidad de la Laguna -Tenerife- & $\begin{array}{l}\text { Facultad de Ciencias Políticas, Humanas } \\
\text { y de la Comunicación }\end{array}$ \\
\hline
\end{tabular}




\begin{tabular}{|l|l|l|}
\hline \multicolumn{2}{|c|}{ Con título de grado en trabajo social } & \multicolumn{1}{|c|}{ Centro directivo } \\
\hline 23 & Universidad de la Rioja & Facultad de Ciencias Jurídicas y Sociales \\
\hline 24 & $\begin{array}{l}\text { Universidad de Las Palmas de Gran } \\
\text { Canaria }\end{array}$ & Facultad de Ciencias Jurídicas \\
\hline 25 & Universidad de León & Escuela Universitaria de Trabajo Social \\
\hline 26 & Universidad de Lleida & $\begin{array}{l}\text { Facultad de Educación, Psicología y } \\
\text { Trabajo Social }\end{array}$ \\
\hline 27 & $\begin{array}{l}\text { Universidad Nacional de Educación a } \\
\text { Distancia (Uned) }\end{array}$ & Facultad de Derecho \\
\hline 28 & Universidad de Oviedo & $\begin{array}{l}\text { Facultad de Comercio, Turismo y } \\
\text { Ciencias Sociales "Jovellanos" }\end{array}$ \\
\hline 29 & Universidad de País Vasco & Escuela Universitaria de Trabajo Social \\
\hline 30 & $\begin{array}{l}\text { * Universidad Pontificia Comillas - } \\
\text { Madrid- }\end{array}$ & $\begin{array}{l}\text { Facultad de Ciencias Humanas y } \\
\text { Sociales }\end{array}$ \\
\hline 31 & Universidad Pública de Navarra & $\begin{array}{l}\text { Facultad de Ciencias Humanas y } \\
\text { Sociales }\end{array}$ \\
\hline 32 & * Universidad Ramón Llul -Barcelona- & $\begin{array}{l}\text { Facultad de Educación Social y Trabajo } \\
\text { Social "Pere Tarrés" }\end{array}$ \\
\hline 33 & Universidad Rovira I Virgili -Tarragona- & Facultad de Ciencias Jurídicas \\
\hline 34 & Universidad de Salamanca & Facultad de Ciencias Sociales \\
\hline 35 & Universidad de Santiago de Compostela & Escuela Universitaria de Trabajo Social \\
\hline 36 & Universidad de Valencia & Facultad de Ciencias Sociales \\
\hline 37 & Universidad de Valladolid & Facultad Educación y Trabajo Social \\
\hline 38 & * Universidad de Vic -Barcelona- & $\begin{array}{l}\text { Facultad de Ciencias de la Salud y el } \\
\text { Bienestar }\end{array}$ \\
\hline 39 & Universidad de Vigo -Orense- & Facultad de Ciencias de la Educación \\
\hline
\end{tabular}

${ }^{*}$ Universidades de titularidad privada.

Fuente: elaboración propia a partir del análisis de las páginas web oficiales de las universidades mencionadas, identificadas en bibliografía cada una de ellas.

\section{Proceso de implantación del título de grado en Trabajo Social}

En el 2008 la titulación se implanta en las Universidades de Zaragoza y "Pontificia Comillas" de Madrid. En el período 2009-2010, 36 universidades (92\%) lo ponen en marcha, siendo el País Vasco la última en su implantación, en el 2011. Los resultados de la investigación muestran que es en 2008 cuando se inició la implantación del Título de Grado en Trabajo Social en España conforme a Bolonia, proceso que ha durado hasta el 2011. Desde el año 2011 no consta que se haya implantado en otro centro docente el Título de Grado en Trabajo Social en España. 


\section{Estructura y organización de las asignaturas vinculadas con la dependencia}

Tras el análisis de las asignaturas de la titulación en las diferentes universidades a través de las guías docentes se constata que son 32 las universidades que imparten asignaturas cuya denominación integra la nomenclatura "dependencia" y, por tanto, directamente relacionados con el objeto de estudio $(82,05 \%)$. De esta forma, de las 39 universidades identificadas y analizadas, 7 de ellas no integran la enseñanza de la dependencia como asignatura específica con tal denominación en su Plan de Estudios (concretamente "Rovira y Virgili" de Tarragona, País Vasco, Santiago de Compostela, Valencia, Valladolid y las privadas Vic y "Ramón LLul", ambas de Barcelona). Ello no significa que en sus planes de estudio no aborden la dependencia en sus asignaturas, sino que ésta es desarrollada desde una perspectiva relacionada con colectivos o sistemas de protección específicos. Así, por ejemplo, la Universidad "Rovira y Virgili" tiene dos asignaturas denominadas "Trabajo Social y Vejez" y "Trabajo $^{2}$ Social y Discapacidad"4, donde tratan, entre otros, la dependencia, pero no son asignaturas que aborden la misma de una manera integrada y transversal y, por tanto, no son unidades de análisis conforme al diseño de investigación previsto.

\section{Asignaturas especifficas de dependencia}

De las 32 universidades que integran la dependencia como asignatura específica, 11 la incorporan en dos asignaturas dentro de su Plan de Estudios. De esta manera, son un total de 43 asignaturas relacionadas directamente con la dependencia, correspondientes a 32 universidades (ver anexo II).

Para el análisis de las asignaturas, se han agrupado en 17 nomenclaturas diferentes y la denominación mayoritaria es "Salud, Dependencia y Vulnerabilidad Social", que representa un 27,9\% del total de 43 asignaturas analizadas. En segundo lugar, la nomenclatura más repetida ha sido "Salud Pública y Trabajo Social" con un 14\%, lo que suma entre las dos primeras casi un $42 \%$ del total analizado. De las 17 nomenclaturas, 5 de ellas no contienen el término de dependencia, y de estas cinco, tres contienen el término "salud".

En relación a la materia donde se integra la asignatura, la denominación más común es: "salud, dependencia y vulnerabilidad social", en coincidencia con lo establecido en el Libro blanco (Aneca, 2004). Se constata, asimismo, que la mayoría de las universidades coinciden en definir la asignatura de dependencia igual que la materia donde se integra.

3 https://moodle.urv.cat/docnet/guia docent/index.php?centre=15\&ensenyament=1522\&assignatura=15224 206\&fitxa _ apartat $=57 \&$ any _ academic $=2016$ _ 17\&idioma $=$ cast\&any _ academic $=2016$ _ 17

4 https://moodle.urv.cat/docnet/guia _ docent/index.php?centre=15\&ensenyament=1522\&consulta=assignat ures\&idioma $=$ cast 
Atendiendo a su carácter, 30 (70\%) son obligatorias y 13 (30\%) optativas. Respecto a las universidades que imparten la asignatura con carácter optativo (13) indicar que en 9 casos las universidades, además de la optativa, ofrecen a su vez una asignatura obligatoria en dependencia y en 4 casos las universidades solo disponen de una asignatura con carácter optativo (Complutense de Madrid, La Rioja, Salamanca y "Pontificia Comillas" de Madrid). En suma en 28 universidades se imparte la asignatura de manera obligatoria (87,5\%) y en 4 de manera optativa (12,5\%). Destacar que de las 28 universidades donde se imparten la asignatura de manera obligatoria, hay 2 (Almería y UNED) donde se ofrecen dos asignaturas especificas de dependencia y ambas son de carácter obligatorio..

\section{Integración de la asignatura en el itinerario formativo}

En relación con la integración de la asignatura de dependencia en el itinerario formativo o curricular de la titulación de trabajo social, señalar, como se muestra en la tabla (2), que mayoritariamente se ofrece en el $3 .^{\circ}$ curso (53,8\% y 23 asignaturas). Por otra parte, existen 4 universidades donde la asignatura se ofrece de manera optativa al alumnado de $3 .^{\circ}$ y $4 .^{\circ}$ curso y, en un caso se no se asigna a ningún curso específico lo que nos hace presuponer que el alumnado puede escogerla en cualquier curso de la titulación. .

Tabla 2. Integración de la asignatura en el itinerario formativo

\begin{tabular}{|l|l|c|c|}
\hline \multicolumn{2}{|l|}{} & $\begin{array}{r}\text { Frecuencia } \\
N .^{\circ} \text { asignaturas }\end{array}$ & Porcentaje \\
\hline \multirow{4}{*}{ Válido } & Curso 2. & 6 & $14,0 \%$ \\
\cline { 2 - 4 } & Curso 3. $^{\circ}$ & 23 & $53,5 \%$ \\
\cline { 2 - 4 } & Curso 3.० y 4. $^{\circ}$ & 4 & $9,3 \%$ \\
\cline { 2 - 4 } & Curso 4. & 9 & $20,9 \%$ \\
\cline { 2 - 4 } & Sin asignar curso & 1 & $2,3 \%$ \\
\cline { 2 - 4 } & Total & 43 & $100,0 \%$ \\
\hline
\end{tabular}

Fuente: elaboración propia resultado de la investigación.

\section{Duración de las asignaturas (ECTS)}

Respecto al número de créditos de la asignatura de dependencia se observa que oscila entre 4 y 6 ECTS, siendo en la mayoría de los casos de 6 ECTS (83,7\% de los casos). 


\section{Análisis de las competencias de las asignaturas especificas de dependencia}

Del análisis de las guías docentes de los planes de estudio de las 43 asignaturas de las 32 universidades que imparten asignatura específica de dependencia se han identificado un total de 216 competencias específicas, objetivos o resultados de aprendizaje. Tras el análisis de contenido de las mismas se han agrupado en función de la dimensión de coincidencia semántica un total de 93 competencias, procediendo a un análisis comparativo en razón de su contenido y vinculación profesional.

\section{a) Competencias agrupadas por tipologías Libro blanco de la titulación}

El Libro blanco título de grado de trabajo social (Aneca, 2004) define un total de 25 competencias específicas de formación disciplinar y profesional propias del trabajo social. Estas competencias han sido clasificadas en seis grandes grupos según la naturaleza de las mismas.

- Grupo 1. Capacidad para trabajar y valorar de manera conjunta con personas, familias, grupos, organizaciones y comunidades sus necesidades y circunstancias.

- Grupo 2. Capacidad para planificar, implementar, revisar y evaluar la práctica del trabajo social con personas, familias, grupos, organizaciones, comunidades y con otros profesionales.

- Grupo 3. Capacidad para apoyar a las personas para que sean capaces de manifestar las necesidades, puntos de vista y circunstancias.

- Grupo 4. Capacidad para actuar en la resolución de las situaciones de riesgo con las personas así como para las propias y las de los colegas de profesión.

- Grupo 5. Capacidad para administrar y ser responsable, con supervisión y apoyo, de la propia práctica dentro de la organización.

- Grupo 6. Capacidad para demostrar competencia profesional en el ejercicio del trabajo social (Aneca, 2004, pp. 187-198).

Conforme se muestra en la tabla 3, algunos centros docentes coinciden con algunas de las 25 competencias específicas que contemplan estos grupos; se encuentra, en todo caso, coincidencia de los seis grupos de competencias generales que cita el Libro blanco (Aneca, 2014). Destacar que el grupo de competencias 2 es el más reconocido en las universidades como competencia descrita en las guías docentes de las asignaturas de dependencia, concretamente en 19 universidades (59,4\%), seguido del grupo 1 de competencias con 14 universidades $(43,7 \%)$. 
Tabla 3. Competencias de las universidades españolas que integran la asignatura específica de "dependencia" que coinciden con las descritas por el Libro blanco de la Aneca

\begin{tabular}{|l|c|c|c|}
\hline \multicolumn{1}{|c|}{$\begin{array}{c}\text { Competencia Libro blanco } \\
\text { (Aneca, 2004, pp. 187-198) }\end{array}$} & $\begin{array}{c}N^{\circ} \\
\text { universidades } \\
\text { integran } \\
\text { competencia }\end{array}$ & $\begin{array}{c}N^{\circ} \\
\text { universidades } \\
\text { no integran } \\
\text { competencia }\end{array}$ & $\begin{array}{c}\text { Total } \\
\text { universidades }\end{array}$ \\
\hline $\begin{array}{l}\text { Grupo 1. Capacidad para trabajar y } \\
\text { valorar de manera conjunta con (..) }\end{array}$ & $14(43,7 \%)$ & $18(56,3 \%)$ & $32(100 \%)$ \\
\hline $\begin{array}{l}\text { Grupo 2.- Capacidad para planificar, } \\
\text { implementar, revisar y evaluar la } \\
\text { práctica del trabajo social con (..) }\end{array}$ & $19(59,4 \%)$ & $13(40,6 \%)$ & $32(100 \%)$ \\
\hline $\begin{array}{l}\text { Grupo 3.- Capacidad para apoyar a } \\
\text { las personas para que sean capaces } \\
\text { de (...) }\end{array}$ & $7(21,9 \%)$ & $25(78,1 \%)$ & $32(100 \%)$ \\
\hline $\begin{array}{l}\text { Grupo 4.- Capacidad para actuar en } \\
\text { la resolución de las situaciones de } \\
\text { riesgo (...) }\end{array}$ & $10(31,2 \%)$ & $22(68,8 \%)$ & $32(100 \%)$ \\
\hline $\begin{array}{l}\text { Grupo 5.- Capacidad para adminis- } \\
\text { trar y ser responsable, (...) }\end{array}$ & $8(25 \%)$ & $24(75 \%)$ & $32(100 \%)$ \\
\hline $\begin{array}{l}\text { Grupo 6.- Capacidad para demostrar } \\
\text { competencia profesional (...) }\end{array}$ & $5(15,6 \%)$ & $27(84,4 \%)$ & $32 \%)$ \\
\hline
\end{tabular}

Fuente: elaboración propia resultado de la investigación.

\section{b) Competencias coincidentes con el documento de referencia nacional "Criterios para el diseño de planes de estudios de títulos de grado en Trabajo Social"}

En el documento marco "Criterios para el diseño de planes de estudios de títulos de grado en Trabajo Social" (Conferencia de Directores de Centros y Departamentos de Trabajo Social y Consejo General de Trabajo Social, 2007), se describen los contenidos que deben tener las asignaturas o módulos de los planes de estudios conducentes a títulos oficiales de graduado en Trabajo Social cuya superación tenga como resultado la adquisición de las competencias que describe el Libro blanco del título de grado en Trabajo Social de la Aneca.

Concretamente la asignatura de dependencia se clasificaría en la materia denominada: Salud, Dependencia y Vulnerabilidad Social, con unos créditos que oscilan entre 5 y 7, quedando establecidas en tres competencias específicas, que concretamente son las siguientes:

C3.1. Conoce y comprende las relaciones entre la salud y la situación social de las personas, el sistema de género, la cultura y el ciclo vital. 
C3.2. Es capaz de identificar y valorar las necesidades sociales relacionadas con los problemas de salud y su repercusión en la vida de las personas afectadas, y las estrategias de atención y apoyo pertinentes en cada situación.

C3.3. Es capaz de reconocer las características de los problemas de salud que dan lugar a necesidades de atención y apoyo sociales, con especial referencia a las discapacidades, a la dependencia, a los problemas de salud mental y a las adicciones (Conferencia de Directores de Centros y Departamentos de Trabajo Social y Consejo General de Trabajo Social, 2007, p.16).

Las competencias mencionadas son escasamente recogidas por las guías docentes de la asignatura de dependencia en las universidades españolas. Concretamente se identifica una de las competencias anteriores en 2 universidades $(6,2 \%)$ donde se imparten la asignatura específica de dependencia.

Tabla 4. Competencias de las guías docentes que coinciden con las descritas en los "Criterios para el diseño de planes de estudios de títulos de grado de Trabajo Social"

\begin{tabular}{|l|c|c|c|}
\hline $\begin{array}{c}\text { Competencia criterios para el diseño de } \\
\text { planes de estudios de títulos de grado } \\
\text { en Trabajo Social (2007: p.16) }\end{array}$ & $\begin{array}{c}N^{\circ} \\
\text { universidades } \\
\text { integran } \\
\text { competencia }\end{array}$ & $\begin{array}{c}N^{\circ} \\
\text { universidades } \\
\text { no integran } \\
\text { competencia }\end{array}$ & $\begin{array}{c}\text { Total } \\
\text { universidades }\end{array}$ \\
\hline $\begin{array}{l}\text { C3.1. Conoce y comprende las rela- } \\
\text { ciones entre la salud y la situación } \\
\text { social (...). }\end{array}$ & $5(15,6 \%)$ & $27(84,4 \%)$ & $32(100 \%)$ \\
\hline $\begin{array}{l}\text { C3.2. Es capaz de identificar y valo- } \\
\text { rar las necesidades sociales relacio- } \\
\text { nadas con los problemas de salud y } \\
\text { (...). }\end{array}$ & $2(6,2 \%)$ & $30(93,8 \%)$ & $32(100 \%)$ \\
\hline $\begin{array}{l}\text { C3.3. Es capaz de reconocer las } \\
\text { características de los problemas de } \\
\text { salud que, (...). }\end{array}$ & $4(12,5 \%)$ & $28(87,5 \%)$ & $32(100 \%)$ \\
\hline
\end{tabular}

Fuente: elaboración propia resultado de la investigación

\section{c) Competencias agrupadas por tipologías vinculadas con competencias profesionales}

El resto de competencias, objetivos o resultados de aprendizaje que se recogen en las distintas universidades en las guías docentes de sus asignaturas se ha procedido a agruparlas en tipologías propias contemplándolas según materia y contenido, identificando categorías necesarias para la profesión del trabajo social. Fruto del análisis de contenido propio de la investigación, se han establecido cuatro tipologías con base en los descriptores identificados y motivados por ser materias imprescindibles en el desarrollo de intervención 
del trabajo social:

- marco teórico/normativo;

- principios éticos, habilidades y deontología del trabajo social;

- metodología del trabajo social y

- acción indirecta.

Cada descriptor identificado se ha recogido en la tipología descrita en relación con su contenido y significado, resultando así:

\section{En relación con el marco teórico / normativo}

En esta tipología se recogen las competencias, habilidades y conocimientos identificados en la materia específica de dependencias relacionadas con el marco teórico, el desarrollo normativo / legislativo y los conocimientos generales del trabajo social en dependencia. Se trata de un conjunto tipológico de competencias específicas cuyo fin es proporcionar al alumnado el conocimiento básico para el desarrollo profesional en el ámbito de la dependencia.

1. Que los estudiantes demuestren poseer y comprender conocimientos en el área de las Ciencias Sociales a un nivel que se apoye en libros de texto avanzados y que implique conocimientos procedentes de la vanguardia del campo de estudio.

2. Establecer valoraciones sociales precisas en casos de dependencia en el marco del Sistema de Promoción de la Autonomía y Atención a la Dependencia.

3. Localizar e identificar y manejar las fuentes disponibles. Conocer e identificar los instrumentos de valoración de las situaciones de dependencia, sus objetivos y su utilidad.

4. Conocer las estrategias de atención y apoyo vinculadas a las situaciones de dependencia y discapacidad. Identificar los rasgos de los modelos de intervención.

5. Conseguir los conocimientos necesarios para intervenir con personas en situación de dependencia y con familias, conocer los recursos y los programas para mejorar la calidad de vida de estas personas.

6. Exponer y dar cuenta de las situaciones de dependencia y su incidencia social. 
7. Conocer y comprender el marco jurídico, económico, político, administrativo y social del nuevo sistema de protección social que nace con la LAAD.

8. Conocer las dimensiones de análisis de la aplicación de la Ley de Autonomía y Dependencia, distinguiendo entre principios teórico-normativos, instituciones y procesos.

9. Conocer el origen y desarrollo de la Ley de Dependencia en España.

10. Conocer el encaje de la dependencia en las leyes de servicios sociales.

11. Conocer las dimensiones de análisis de los servicios sociales y el sistema para la autonomía y atención a la dependencia, así como su evolución y desarrollo.

12. Conocer la estructura administrativa que posibilita la ejecución de las prestaciones que se derivan de la Ley de Dependencia.

13. Competencia para adquirir e integrar los avances legales y reglamentarios en materia social, sociosanitaria y socioeducativa en la actividad del trabajador social.

14. Documentarse, actualizar y profundizar en los conocimientos propios relacionados con el trabajo social.

15. Aprender a manejar normativa referencial.

16. Identificar los servicios, recursos y ayudas existentes.

17. Conocer los elementos diferenciales tales como la etnia, la cultura y el género y su influencia en las situaciones de discriminación, opresión y vulnerabilidad, materializadas en el diferente acceso a los recursos de las personas, grupos y comunidades.

18. Conocer el funcionamiento de los programas de acción social de la Unión Europea y su incidencia en la política de los Estados miembros y los desafíos a los que se enfrentan.

19. Conocer e identificar los contextos sociales en los que se diseñan, prueban y modifican las estrategias de abordaje y gestión de los procesos tipificados como sanitarios.

20. Capacidad para conocer los procesos psicológicos básicos en las diferentes etapas del desarrollo humano.

21. Capacidad para conocer las situaciones de conflicto y desajuste en las diferentes etapas del desarrollo humano. 
22. Reconocer factores psicosociales que intervienen en los procesos de salud / enfermedad que requieren de atención y apoyo de trabajo social.

23. Conocer la importancia que tiene la salud, entendida en su aspecto positivo e integral, las consecuencias de la pérdida de salud e identificar factores de riesgo y comprender las perspectivas sociológicas sobre la dependencia, la salud y la vulnerabilidad social.

24. Capacidad para integrar y aplicar los conocimientos sobre las necesidades humanas y las políticas sociales en el estudio, diagnóstico, planificación, intervención y evaluación de programas dentro de las organizaciones de protección social.

En relación con principios éticos, habilidades y deontología del trabajo social

Esta segunda tipología engloba las competencias relacionadas con las habilidades que el alumnado debe adquirir en relación con los principios éticos y la deontología del trabajo social, en habilidades que deben darse en la práctica profesional. Tratan aspectos generales del trabajo social y no específicos de dependencia, aunque sí relacionados.

1. Que los estudiantes sepan aplicar sus conocimientos a su trabajo o vocación de una forma profesional y posean las competencias que suelen demostrarse por medio de la elaboración y defensa de argumentos y la resolución de problemas dentro de su área de estudio.

2. Que los estudiantes tengan capacidad de reunir e interpretar datos relevantes para emitir juicios que incluyan una reflexión sobre temas importantes de índole social, científica o ética.

3. Que los estudiantes puedan transmitir información, ideas, problemas y soluciones a un público tanto especializado como no.

4. Que los estudiantes hayan desarrollado aquellas habilidades necesarias para emprender estudios posteriores con un alto grado de autonomía.

5. Adaptarse a situaciones nuevas y afrontar situaciones profesionales de forma creativa

6. Elaborar y gestionar los documentos básicos de comunicación.

7. Capacidad para transmitir y potenciar la igualdad de oportunidades, la accesibilidad universal a los derechos humanos, los valores de democracia y paz, y la sostenibilidad.

8. Incorporar y defender los principios y valores éticos relacionados con el respeto y la defensa de los derechos humanos, planteando soluciones a las 
problemáticas de la intervención profesional que contemplan y están basadas en estos valores y principios.

9. Analizar la congruencia entre lo posible y lo real.

En relación con la metodología del trabajo social

En esta tercera tipología se identifican las competencias que el alumnado precisa respecto a la metodología propia del trabajo social, intervención, técnicas e instrumentos a emplear. Esta tipología describe algunas competencias específicas respecto a la materia de dependencia.

1. Trazar estrategias de intervención social ante situaciones de dependencia valoradas.

2. Adaptarse al método general del trabajo social y aplicar sus excepciones dependiendo de los diferentes ámbitos de actuación.

3. Identificar escenarios donde las situaciones de dependencia pueden estar condicionados por factores sociofamiliares.

4. Elaborar planes individuales de atención en casos de personas en situación de dependencia y colectivos en situación de necesidad social.

5. Valorar conjuntamente con los sujetos la situación inicial y decidir la mejor forma de atender y cubrir la demanda y/o necesidad que justifica la intervención del trabajador social.

6. Seleccionar el modelo teórico más adecuado para una intervención eficaz.

7. Respetar las fases y pasos a seguir en los distintos ámbitos de la intervención.

8. Ser capaz de evaluar situaciones de dependencia y analizar la información, teniendo en cuenta los puntos de vista de los participantes, los conceptos teóricos, los datos de la investigación, las normas legales y los procedimientos institucionales, para la elaboración de informes sociales.

9. Analizar la interdependencia de sus miembros y sus relaciones.

10. Ser capaz de aplicar los métodos e instrumentos de valoración de la dependencia y las opciones posibles para establecer las estrategias de intervención social.

11. Ser capaz de reconocer y analizar las problemáticas y necesidades de los diferentes colectivos en situación de dependencia. 
12. Analizar críticamente las relaciones existentes entre desigualdad, pobreza y salud.

13. Aplicar de forma razonada las técnicas en cada fase del método de trabajo empleado.

14. Capacidad de conocer y comprender los principales aspectos de los desequilibrios y desigualdades sociales y los mecanismos de discriminación, así como identificar las situaciones de precariedad, desprotección, segregación, marginación y vulneración de derechos o incumplimiento de responsabilidades sociales que son objetivo de intervención del trabajo social.

15. Comprender los elementos que conforman un plan de intervención sociosanitario desde sus diferentes ámbitos.

16. Establecer el marco de trabajo legal y el procedimiento para acceder a los historiales e informes.

17. Trabajar con las personas, grupos y comunidades en la identificación de sus necesidades y problemas, en la evaluación de los medios disponibles, personales y sociales, para valorar las metodologías de intervención más idóneas.

18. Entender el carácter dinámico y la necesidad de implementación de la intervención socio-sanitaria.

19. Evaluar el proceso y los resultados de las actividades desarrolladas.

20. Estudiar las causas de los incumplimientos de los objetivos.

21. Dirigir las acciones individuales, familiares, grupales y comunitarias, motivando la superación de la problemática detectada, mediante el desarrollo de las capacidades y habilidades de los usuarios.

22. Ser capaz de dirigir las acciones individuales, familiares, grupales y comunitarias, motivando la autogestión y la autonomía.

23. Capacidad para aplicar de forma adecuada los recursos de los sistemas públicos de protección social a las distintas situaciones de necesidad.

24. Obtener las competencias necesarias para la valoración y programación de los distintos servicios y prestaciones que deberán utilizarse.

25. Ser capaz de diseñar una intervención individual, familiar, grupal y / o colectiva según el nivel asistencial de salud.

En relación con acción indirecta 
Esta cuarta tipología recoge las competencias de acción indirecta en relación con la prevención, promoción, coordinación y/o trabajo en equipo que se da en el trabajo social y que desde la práctica profesional es necesario para el desarrollo de cualquier intervención profesional. Presenta descriptores específicos en materia de dependencia.

1. Aprender a identificar entornos familiares e institucionales adecuados a las nuevas demandas de las personas dependientes y gestionar las estrategias sociales pertinentes, primando los criterios de prevención y eficacia.

2. Establecer propuestas para una adecuada formación y cualificación de profesionales y cuidadores en la Ley de Promoción de la Autonomía Personal y Atención a las Personas en Situación de Dependencia.

3. Planificar, instrumentar, revisar y evaluar la práctica del trabajo social con personas, grupos, familias, organizaciones, comunidades y otros profesionales.

4. Determinar el marco de intervención de cada profesional.

5. Conocer e identificar las estrategias de atención y apoyo pertinentes a cada situación. Conocer los diferentes dispositivos formales e informales de atención y sus diferencias.

6. Apoyar y defender a las personas para promover la integración social, la autonomía personal, la cohesión y el bienestar social, contribuyendo a la ciudadanía activa mediante el empowerment y la garantía de los derechos humanos y sociales, la igualdad de oportunidades y accesibilidad entre todas las personas, el respeto a la diversidad y la convivencia democrática.

7. Distinguir y plantear problemas abordables mediante políticas sociales.

8. Favorecer las actuaciones profesionales de prevención primaria y secundaria, evaluando las situaciones.

9. Contribuir a la administración de recursos y servicios colaborando con los procedimientos implicados en su obtención, supervisando su eficacia y asegurando su calidad.

10. Ser capaz de identificar las actitudes, los estereotipos y prejuicios sociales, sus mecanismos de formación y cambio, y su influencia en la identidad personal y social para poder aplicarlos a la prevención y diagnóstico de las necesidades sociales de la ciudadanía en situación de riesgo de discriminación y exclusión. 
11. Analiza críticamente la realidad social, como forma de identificar y prevenir la aparición de nuevas problemáticas sociales.

12. Valorar la importancia que tiene la promoción y educación para la salud en el desarrollo de la persona y la comunidad y desarrollar actitudes preventivas en el ejercicio profesional.

13. Adquirir las competencias necesarias para actuar y asesorar en problemas de salud, sociales, en unidades de individuos, familias y ámbitos institucionales con el propósito de anticipar, prevenir y mejorar el nivel de salud y bienestar social.

En definitiva, como muestra la tabla siguiente (5), la mayoría de las universidades hacen referencia al marco teórico / normativo del trabajo social (13 universidades -40,6\%-), mientras que solo 6 universidades relacionan las competencias de la asignatura de dependencia con los principios éticos, habilidades y deontología profesional (18,7\%).

Tabla 5. Competencias de las guías docentes que coinciden con las descritas en categorías según contenido similar

\begin{tabular}{|l|l|l|l|}
\hline \multicolumn{1}{|c|}{$\begin{array}{c}\text { Competencias en categorías según } \\
\text { contenido similar }\end{array}$} & $\begin{array}{c}N_{.^{\circ}} \\
\text { universidades } \\
\text { integran } \\
\text { competencia }\end{array}$ & $\begin{array}{c}N^{\circ} \\
\text { universidades } \\
\text { no integran } \\
\text { competencia }\end{array}$ & \multicolumn{1}{|c|}{$\begin{array}{c}\text { Total } \\
\text { universidades }\end{array}$} \\
\hline Marco teórico / normativo & $13(40,6 \%)$ & $19(59,4 \%)$ & $32(100 \%)$ \\
\hline $\begin{array}{l}\text { Principios éticos, habilidades y } \\
\text { deontología }\end{array}$ & $6(18,7 \%)$ & $26(81,3 \%)$ & $32(100 \%)$ \\
\hline Metodología & $11(34,4 \%)$ & $21(65,6 \%)$ & $32(100 \%)$ \\
\hline Acción indirecta & $8(25 \%)$ & $24(75 \%)$ & $32(100 \%)$ \\
\hline
\end{tabular}

Fuente: elaboración propia resultado de la investigación.

\section{Conclusiones}

La investigación ha permitido identificar los centros docentes de enseñanza superior de España donde se imparte el título de grado en Trabajo Social, concretamente 39 universidades (48,14\% de las universidades españolas). La implantación de los títulos de Trabajo Social como grados se produce mayoritariamente en el curso académico 2009-2010. El análisis de contenido de las guías docentes del conjunto de asignaturas de todos los títulos de grado en Trabajo Social de España ha permitido identificar 43 asignaturas que tratan la materia de dependencia de manera específica y transversal en un total de 32 
universidades. Cabe destacar que 7 universidades, a pesar de la promulgación de la Ley 39/2006 de 14 de diciembre de Promoción de la Autonomía Personal y Atención a las personas en situación de dependencia, anterior a la implantación del título, no contemplan en su plan de estudios asignatura específica de dependencia. A pesar de lo anterior, no debe inducirse que no aborden el tema de la dependencia en otras asignaturas no específicas.

En cuanto a la denominación de la asignatura se identifican 17 nomenclaturas diferentes con una duración de 6 ECTS y la mayoría coincide con el nombre de la materia: Salud, Dependencia y Vulnerabilidad Social como se indica en el documento de referencia "Criterios para el diseño de planes de estudios de títulos de grado en Trabajo Social". Sin embargo, también se observa el uso del concepto de discapacidad ligado a la nomenclatura de la asignatura con dependencia. Se considera necesaria una distinción entre ambos conceptos dado que, aunque pueden encontrarse relacionados, no tienen un mismo significado conceptual ni presentan marcos normativos y de intervención idénticos (por ejemplo, una persona puede presentar discapacidad, pero no encontrarse en una situación de dependencia).

Otro aspecto a resaltar es el curso donde se imparte la asignatura de dependencia, que en la mayoría de las universidades está entre $3 .^{\circ}$ y $4 .^{\circ}$ curso. Esto puede resultar desventajoso al alumnado que realiza las prácticas preprofesionales en ámbitos de intervención relacionados con atención a personas en situación de dependencia, dado que no han adquirido los conocimientos, habilidades y competencias específicos para la adecuada comprensión del desempeño profesional.

En cuanto al análisis de contenido de las competencias específicas cabe indicar que es donde se ha encontrado mayor dificultad de análisis. Por una parte, destacar la dificultad de acceso y análisis de la información de las guías docentes, donde, además, se ha constatado el empleo de diferentes denominaciones: competencias, objetivos o resultados de aprendizaje para hacer referencia a lo mismo (destrezas y habilidades que debe adquirir el alumnado). Por el otro, la complejidad de las numerosas y distintas denominaciones de estas utilizadas por las universidades, adoptando el criterio de agruparlas por tipología según contenido similar, dado que "las competencias no pueden dejar de lado los contenidos" (Escudero, 2008, p. 9).

La investigación muestra que las competencias que recogen las universidades en sus respectivas guías docentes de las asignaturas vinculadas con dependencia coinciden mayoritariamente con las descritas por el Libro blanco título de grado de Trabajo Social (Aneca, 2004), valorándose, por tanto, competencias más generales que propias de la materia. Seguidamente, las más indicadas son las 
establecidas en la tipología clasificada como marco teórico/normativo, donde se engloban competencias más específicas de la materia que ocupa la asignatura de la dependencia. Se considera esta categoría fundamental donde se relacionan contenidos específicos e iniciales de la materia para vincular las competencias que debe alcanzar el alumnado en asignaturas de dependencia. Sin embargo, son las competencias en materia de Salud, Dependencia y Vulnerabilidad Social que describe el documento marco "Criterios para el diseño de planes de estudios de títulos de grado en Trabajo Social" las menos indicadas por las universidades en sus guías docentes, a pesar de ser específicas de dependencia.

La docencia incluye una función pedagógica que va más allá de proveer conocimientos: ha de garantizar la adquisición de competencias que capacitarán a los egresados en la toma de decisiones con respecto a tareas propias de su actividad profesional (Touriñán, 2013). De los resultados obtenidos, podemos concluir con algunas consideraciones propositivas o recomendaciones en la enseñanza de la dependencia de los centros docentes en los títulos de grado en Trabajo Social; concretamente:

- Introducir la asignatura en materia de dependencia antes del inicio de las prácticas pre-profesionales con el fin de que el alumnado llegue a su ámbito profesional de prácticas con conocimientos en esta materia. Se trata de vincular las prácticas profesionales con las competencias específicas adquiridas en el ámbito de actuación donde se desarrollen las prácticas.

- Valorar los contenidos que deben integrar las asignaturas según materia a tratar, intentando dar mayor coherencia teórica, normativa y práctica en la especificidad del tema que ocupe.

- Indicar en las guías docentes, objetivos o resultados de aprendizaje más específicos de dependencia, tal y como se recogen en las tipologías marco teórico/normativo y metodología del trabajo social, donde se recogen descriptores más específicos de la materia de dependencia.

- Tratar de unificar criterios en la enseñanza de la dependencia en los títulos de grado de Trabajo Social en los centros docentes de España.

\section{Referentes hibliográficos}

Agencia Nacional de Evaluación de la Calidad y Acreditación (Aneca) (2004). Libro blanco título de grado de Trabajo Social. Madrid: Aneca.

Caparros, N. y Raya E. (2014). La supervisión en las prácticas de grado de Trabajo Social: acompañamiento en la intervención social. En Pastor, E. (Ed.). El trabajo social ante el reto de la crisis y la educación superior. Madrid: Universitas, pp. 1133-1140. 
Chaparro Maldonado, M. Y. y Urra Canales, M. (2014). Competencias específicas del trabajador social en la gestión del talento humano. Tendencias y Retos, V. 19, n. ${ }^{\circ}$ 2, pp. 27-44.

Conferencia de Directores y Directoras de Centros y Departamentos de Trabajo Social y Consejo General de Colegios Oficiales de Diplomados en Trabajo Social (2007). La formación universitaria en Trabajo Social. Criterios para el diseño de planes de estudios de títulos de Grado en Trabajo Social. Barcelona.

De Juana Oliva, A. y Fernández Lozano, M. P. (2008). Competencias y estrategias de aprendizaje. Reflexiones sobre el proceso de cambio en el EEES. Cuadernos de Trabajo Social, n. ${ }^{\circ} 21$, pp. 217-230.

Echeverría, B. (2003). Saber y sabor de la profesionalidad. Revista de Formación y Empleo, V. 74, pp. 6-11

España. Ley 39/2006 de 14 de diciembre, de Promoción de la Autonomía Personal y Atención a las personas en situación de dependencia. Publicado en Boletín Oficial del Estado núm. 299, de 15 de diciembre de 2006. 44142-44156.

España. Marco Español de Cualificaciones (MECU). En: http://www.mecd.gob.es/educacion-mecd/ $\mathrm{mc} / \mathrm{mecu} /$ preguntas-frecuentes-glosario.html (consultado el 17/10/2016).

España. Real Decreto 22/2015, de 23 de enero, por el que se establecen los requisitos de expedición del Suplemento europeo a los títulos regulados en el Real Decreto 1393/2007, de 29 de octubre, por el que se establece la ordenación de las enseñanzas universitarias oficiales y se modifica el Real Decreto 1027/2011, de 15 de julio, por el que se establece el Marco Español de Cualificaciones para la Educación Superior. Publicado en Boletín Oficial del Estado núm. 33, de 7 de febrero de 2015, 10325-10336.

Gómez, F. (2010). Competencias profesionales en trabajo social. Portularia, V. 10 n. 2 2, pp. 51-63.

Ministerio de Educación, Cultura y Deporte (2015). Datos y cifras del Sistema Universitario. Madrid.

Pastor Seller, E. (2014). Implicaciones del Espacio Europeo de Educación Superior para el Trabajo Social en España. Revista Lugares de Educação, V. 4, n. 9, pp. 163-181.

Pastor Seller, E., Martínez, B. y Torralba, R. (2016). Prácticas profesionales y competencias en Trabajo Social con grupos y comunidades. Interacción y Perspectiva, V. 6, n. ${ }^{\circ}$ 2, pp. 129-143.

Rodríguez, A. (2015). La formación universitaria en competencias: incidencia en la evaluación pedagógica de los procesos de enseñanza aprendizaje desde el enfoque socioformativo. Humanismo y Trabajo Social, V. 15. n. 1 , pp. 33-45.

Rodríguez González, R. (2006). Diseño de entornos para el desarrollo de la autonomía del aprendizaje. Aula Abierta, n. ${ }^{\circ}$ 87, pp. 89-104.

Touriñán López, J. M. (2013). El significado de la función pedagógica y la necesidad de generar principios de acción. Revista Española de Pedagogía, V. 254, pp. 29-47.

Universia (2010). ¿Qué es una guía docente? Portal de las universidades españolas. En: http://noticias. universia.es/ciencia-nn-tt/reportaje/2010/05/03/647368/1/guia-docente-esqueleto-asignatura/guiadocente.html (consultado el 17/10/2016).

Universidad de Alicante (2010). Orientaciones para la elaboración de guías docentes. Alicante: Instituto de Ciencias de la Educación. En: http://web.ua.es/es/ice/documentos/recursos/materiales/guia-docente. pdf (consultado el 17/10/2016).

Universidad de Alicante (2016). Grado en Trabajo Social. Alicante. En: http://cv1.cpd.ua.es/ConsPlanesEstudio/cvFichaAsiEEES.asp?wCodEst $=$ C155\&wcodasi $=19513 \&$ wLengua $=$ C\&scaca=2015-16 (consultado el 09/06/2016). 
Universidad de Almería (2016). Grado en Trabajo Social. Almería. En: http://cms.ual.es/UAL/estudios/ grados/plandeestudios/asignaturas/asignatura/GRAD06810?idAss $=68103220 \& \mathrm{idTit}=6810 \& \mathrm{an}$ yo _ actual=2015-16 (consultado el 08/06/2016);

Universidad de Barcelona (2016). Grado en Trabajo Social. Barcelona. En: http://www.ub.edu/dyn/cms/ print/p.jsp?u=/continguts_es/estudis/oferta _ formativa/graus/fitxa/T/G1027/index.html (consultado el 09/02/2016);

Universidad de Castilla La Mancha (2016). Grado en Trabajo Social. Cuenca. En: https://guiae.uclm. es/vistaPrevia/22768/999 (consultado el 08/06/2016); http://trabajosocialencuenca.blogspot.com. es/2014/07/nueva-definicion-global-de-trabajo.html (consultado el 08/06/2016).

Universidad de Castilla La Mancha (2016). Grado en Trabajo Social. Talavera de la Reina. En: https:// guiae.uclm.es/vistaPrevia/22402/999 (consultado el 09/06/2016); https://guiae.uclm.es/vistaPrevia/21775/999 (consultado el 09/06/2016).

Universidad de Complutense de Madrid (2016). Grado en Trabajo Social. Madrid. En: http://trabajosocial. ucm.es/estudios/grado-trabajosocial-planestudios (consultado el 08/06/2016); http://trabajosocial.ucm. es/data/cont/docs/6-2013-05-28-DEPENDENCIA\%20ENVEJECIMIENTO\%20Y\%20DISCAPACIDAD26. pdf (consultado el 08/06/2016).

Universidad de Cádiz (2016). Grado en Trabajo Social. Jerez. En: http://asignaturas.uca.es/wuca _ asignaturasttg1516 _ asignatura?titul=30406\&asign=30406026\&dpto=C112 (consultado el 08/06/2016).

Universidad de Deusto (2016). Grado en Trabajo Social. Bilbao. En: http://www.deusto.es/cs/Satellite/ deusto/es/nuevos-estudiantes-de-grado/estudios-grados/trabajo-social-campus-bilbao/programa?i dPest=1336641263314\&idSeccion=1 (consultado el 08/06/2016); http://ies.duquederivas.rivas.educa. madrid.org/duque/images/stories/orienta10_11/infouniversidades/html/CT/UniversidaddeDeusto. htm (consultado el 08/06/2016); http://repstud.deusto.es/reports/rwservlet?gur01008a\&par cgacocea $=143 \&$ par _ cgacosea $=4 \&$ par _ asignatura _ codigo $=43425 \&$ par _ gua _ curso $=201516 \&$ par _ guo _ codigo=1\&par_destino=WEBPUBLICA\&par _ idioma _ web=SPSP\& _ ga $=1.59870441 .51096763$ 3.1455908500 (consultado el 08/06/2016); http://repstud.deusto.es/reports/rwservlet?gur01008a\&par cgacocea $=143 \&$ par _ cgacosea $=4 \&$ par _ asignatura _ codigo $=43445 \&$ par _ gua _ curso $=201516 \&$ par guo _ codigo $=1 \& \mathrm{par}$ _ destino $=$ WEBPUBLICA\&par _ idioma _ web $=$ SPSP\& _ ga $=1.56782062 .5109676$ 33.1455908500 (consultado el 08/06/2016).

Universidad de Deusto (2016). Grado en Trabajo Social. San Sebastián. En: http://www.deusto.es/cs/ Satellite/deusto/es/nuevos-estudiantes-de-grado/estudios-grados/trabajo-social-campus-bilbao/prog rama?idPest=1336641263314\&idSeccion=1 (consultado el 08/06/2016); http://ies.duquederivas.rivas. educa.madrid.org/duque/images/stories/orienta10_ 11/infouniversidades/html/CT/UniversidaddeDeusto.htm (consultado el 08/06/2016); http://repstud.deusto.es/reports/rwservlet?gur01008a\&par cgacocea $=243 \&$ par _ cgacosea $=4 \&$ par _ asignatura _ codigo $=53425 \&$ par _ gua _ curso $=201516 \&$ par guo _ codigo=1\&par _ destino=WEBPUBLICA\&par _ idioma _ web=SPSP\& _ ga $=1.28009976 .51096763$ 3.1455908500 (consultado el 08/06/2016); http://repstud.deusto.es/reports/rwservlet?gur01008a\&par cgacocea $=243 \&$ par _ cgacosea $=4 \&$ par_ asignatura _ codigo $=53434 \&$ par _ gua _ curso $=201516 \&$ par guo _codigo $=1 \& \mathrm{par}$ _ destino $=$ WEBPUBLICA\&par _ idioma _ web $=$ SPSP\& _ ga $=1.258173262 .510967$ 633.1455908500 (consultado el 08/06/2016).

Universidad de Extremadura (Santa Ana) (2016). Grado en Trabajo Social. Badajoz. En: http://www. universidadsantana.com/grado-en-trabajo-social.html\#planes-de-estudios (consultado el 09/06/2016); http://www.universidadsantana.com/images/grados/trabsocial/programas/Programa _ de _ Trabajo_Social_y_ Dependencia.pdf (consultado el 09/06/2016). 
Universidad de Girona (2016). Grado en Trabajo Social. Girona. En: http://www.udg.edu/tabid/13296/ default.aspx?idpla=3101G0610\&language=es-ES\&anyacad=2015 (consultado el 09/06/2016); http:// www.udg.edu/fep/WebEstudis/TreballSocial/EstudiarTreballSocialalaUdG/tabid/18258/language/ es-ES/Default.aspx (consultado el 09/06/2016); http://www.udg.edu/Guiadematricula/Dissenyassignatura/tabid/15700/Default.aspx?curs=2015\&codia=3101G06019\&codip=3101G0610\&language $=e s-E S$ (consultado el 09/06/2016).

Universidad de Granada (2016). Grado en Trabajo Social. Granada. En: http://grados.ugr.es/trabajosocial/ pages/infoacademica/verificatrabajosocial/! (p.70) (consultado el 08/06/2016); http://trabajosocial. ugr.es/pages/curso-20152016/horariossegundocuatrimestre20152016/!; http://trabajosocial.ugr.es/ pages/info_academica/grado _ trabajo _ social/guiadocentedependencia20132014/! (consultado el 08/06/2016).

Universidad de Huelva (2016). Grado en Trabajo Social. Huelva. En: http://www.uhu.es/etso/sites/ default/files/wfm/guias2015-16/3C/spts.pdf (consultado el 08/06/2016); http://www.uhu.es/etso/sites/ default/files/wfm/guias2015-16/4C/tsdd.pdf (consultado el 08/06/2016).

Universidad de Islas Baleares (2016). Grado en Trabajo Social. Islas Baleares. En: http://estudis.uib.es/ es/grau/treballsocial/GTSO-P/ (consultado el 09/06/2016); http://estudis.uib.es/es/grau/treballsocial/ GTSO-P/21918/index.html (consultado el 09/06/2016).

Universidad de Jaén (2016). Grado en Trabajo Social. Jaén. En: https://uvirtual.ujaen.es/pub/es/informacionacademica/catalogoguiasdocentes/p/2015-16/6/110A/11012019/es/2015-16-11012019 _ es.html (consultado el 08/06/2016); https://uvirtual.ujaen.es/pub/es/informacionacademica/catalogoguiasdocentes/ p/2015-16/6/110A/11013013/es/2015-16-11013013 _ es.html (consultado el 08/06/2016).

Universidad de La Laguna (2016). Grado en Trabajo Social. Tenerife. En: http://www.ull.es/view/centros/ fcps/Guias_docentes/es (consultado el 08/06/2016).

Universidad de La Rioja (2016). Grado en Trabajo Social. La Rioja. En: http://www.unirioja.es/estudios/ grados/trabajo_social/guias.shtml (consultado el 09/06/2016); http:/www.unirioja.es/estudios/grados/ trabajo_social/ (consultado el 09/06/2016).

Universidad de La Salle (2016). Grado en Trabajo Social. Madrid. En: http://www.lasallecentrouniversitario.es/estudios/estudios_ grado/educacion_CC__sociales/Documents/GUIAS\%2013-14/ Gu\%C3\%ADas\%20GTS/3\%C2\%BA\%20TS/GTS-6\%201314\%20SYD.pdf (consultado el 09/06/2016).

Universidad de Las Palmas de Gran Canaria (2016). Grado en Trabajo Social. Las Palmas de Gran Canaria. En: http://www2.ulpgc.es/index.php?pagina=plan _ estudio\&ver=pantalla\&numPantalla=0 3\&codTitulacion=4020\&codPlan=40\&tipotitulacion $=\mathrm{G}$ (consultado el 09/06/2016); http://www2.ulpgc. es/archivos/plan_estudios/4034_40/Memoria _ verifica _ del_grado_ GTS.pdf (p. 129) (consultado el 09/06/2016).

Universidad de León (2016). Grado en Trabajo Social. León. En: https://www.unileon.es/estudiantes/ estudiantes-grado/oferta-de-estudios/grado-en-trabajo-social (consultado el 08/06/2016); http://www4. unileon.es/trabajo_social/guias/1202023 _ 2016.pdf (consultado el 08/06/2016).

Universidad de Lleida (2016): Grado en Trabajo Social. Lleida. En: http://www.treballsocial.udl.cat/ es/index.html (consultado el 20/02/2016); http://guiadocent.udl.cat/pdf/es/101716-1516 (consultado el 08/06/2016).

Universidad de Murcia (2016). Grado en Trabajo Social. Murcia. En: https://aulavirtual.um.es/umugdocente-tool/htmlprint/guia/RJk5PuEL4zudnfaFzZWunf0qhxol71nujgZiekpckNVzvEvvRwy (consultado 
el 08/06/2016); http://www.um.es/web/trabajosocial/contenido/estudios/grados/trabajo-social/2015-16/ guias (consultado el 08/06/2016).

Universidad de Málaga (2016). Grado en Trabajo Social. Málaga. En: https://oas.sci.uma.es:8443/ ht/2015/ProgramasAsignaturas_ Titulacion_5114_AsigUMA_ 52469.pdf (consultado el 08/06/2016).

Universidad de Navarra (2016). Grado en Trabajo Social. Navarra. En: http://www.unavarra.es/fachumanasysociales/estudios/grado/grado-en-trabajo-social/normativa?submenu=yes (consultado el 09/06/2016).

Universidad de Oviedo (2016). Grado en Trabajo Social. Oviedo. En: http://www.unioviedo.es/eutsg/plan. htm (consultado el 20/02/2016); http://sies.uniovi.es/ofe-pod-jsf/web/oferta/seccion-1.faces (consultado el 08/06/2016); http://sies.uniovi.es/ofe-pod-jsf/web/oferta/seccion-5.faces (consultado el 08/06/2016).

Universidad de País Vasco/EHU (2016). Grado en Trabajo Social. País Vasco. En: http://www.ehu.eus/ es/web/gizarte-langintza (consultado el 09/06/2016).

Universidad de Pontificia Comillas (2016). Grado en Trabajo Social. Madrid. En: https://www.educacion. gob.es/ruct/estudio.action?codigoCiclo=SC\&codigoTipo $=$ G\&CodigoEstudio $=2500088 \&$ actual $=$ est udios (consultado el 08/06/2016); http://www.chs.comillas.edu/es/grados/grado-en-trabajo-social-ydiploma-en-cooperacion-internacional-al-desarrollo-opcion-internacional (consultado el 08/06/2016); http://intranet.upcomillas.es/documentacion/documentos/ects/2016/0000008170.pdf (consultado el 08/06/2016).

Universidad de Ramón Llul (2016). Grado en Trabajo Social. Barcelona. En: http://www.peretarres.org/ arxius/facultat/informes/informe _ seguiment _ gts.pdf (consultado el 09/06/2016).

Universidad de Rovira y Virgili (2016). Grado en Trabajo Social. Tarragona. En: http://www.urv.cat/ cae/graus/es_graudetreballsocial.html (consultado el 08/06/2016).

Universidad de Salamanca (2016). Grado en Trabajo Social. Salamanca. En: http://www.usal.es/webusal/ node/2184 (consultado el 08/06/2016).

Universidad de Santiago de Compostela (2016). Grado en Trabajo Social. Santiago de Compostela. En: http://www.usc.es/es/centros/tsocial/titulacions.html?plan=14096\&estudio=14097\&codEstudio=13 $649 \&$ valor $=9$ (consultado el 08/06/2016).

Universidad de Valencia (2016). Grado en Trabajo Social. Valencia. En: http://www.uv.es/uvweb/ universidad/es/estudios-grado/oferta-grados/oferta-grados/grado-trabajo-social-1285846094474/ Titulacio.html?id=1285847315808\&plantilla=UV/Page/TPGDetaill\&p2=2 (consultado el 09/06/2016).

Universidad de Valladolid (2016). Grado en Trabajo Social. Valladolid. En: http://www.uva.es/export/ sites/uva/2.docencia/2.01.grados/2.01.02.ofertaformativagrados/2.01.02.01.alfabetica/Grado-enTrabajo-Social/ (consultado el 08/06/2016).

Universidad de Vic (2016). Grado en Trabajo Social. Barcelona. En: http://www.uvic.cat/es/calendariodextinciondeestudios (consultado el 22/02/2016); http://www.uvic.cat/es/estudi/treball-social (consultado el 08/06/2016).

Universidad de Vigo (2016). Grado en Trabajo Social. Orense. En: http://educacion-ou.webs.uvigo. es/educacion-ou/web/index.php/estudos/2013-08-08-12-51-38/grao-traballo-social (consultado el 08/06/2016).

Universidad de Zaragoza (2016). Grado en Trabajo Social. Zaragoza. En: http://titulaciones.unizar.es/ admin/lectorPDFasig.php?asignatura=26118\&year=2015\&idioma=1 (consultado el 08/06/2016); http:// 
titulaciones.unizar.es/admin/lectorPDFasig.php?asignatura=26154\&year=2015\&idioma=1 (consultado el 08/06/2016).

Universidad Internacional de La Rioja UNIR (2016). Grado en Trabajo Social. La Rioja. En: http://www. unir.net/ciencias-sociales/grado-trabajo-social-online/549200001503/\#-plan-de-estudios (consultado el 08/06/2016).

Universidad Nacional de Educación a Distancia (2016). Grado en Trabajo Social. UNED. En: http://portal. uned.es/portal/page? _ pageid=93,47800945\& _ dad=portal\& _ schema=PORTAL\&idGrado $=6603$ (consultado el 09/06/2016).

Universidad Pablo de Olavide (2016). Grado en Trabajo Social. Sevilla. En: http://www.upo.es/fcs/ contenido?pag=/portal/fcs/alumnos/Grado_ Trabajo_Social\&menuid=\&vE=D72862 (consultado el 08/06/2016); http://www.upo.es/export/portal/com/bin/portal/fcs/alumnos/Guias _ Docentes/GuiasDocentes_2015_2016/GuiasDocentes_GTS/1454938343925_801023_guxa _docente _ salud_pxblica. pdf (consultado el 08/06/2016) ; http://www.upo.es/export/portal/com/bin/portal/fcs/alumnos/Guias Docentes/GuiasDocentes_2015_2016/GuiasDocentes_GTS/1438065134812 _ 801042 _ guxa _ docente _ts_y_autonomxa _ de _las_personas.pdf acceso el 08/06/2016).

Vázquez, O. (2005a). El espacio europeo de educación superior y el trabajo social en España. Portularia, V. 5, n. ${ }^{\circ}$, pp. 239-255.

Vázquez, O. (Coord.) (2005b). Libro blanco del título de grado en Trabajo Social. Madrid: Agencia Nacional de Evaluación y Acreditación de la Calidad (Aneca).

Zabalza, M. A. (2004). Guía para la planificación didáctica de la docencia universitaria en el marco del EEES (Guía de guías). Santiago de Compostela: Universidad Santiago de Compostela. 